Pediatr. Res. 14: 1432-1438 (1980)

\title{
Index to Volume 14
}

Abdellif, M. M. 916

Abnormal cell-mediated immunity 96

Abnormal kinetics 54

Abnormal organic aciduria 1097

Acetylcholine 1311

Acid-base balance 286

Acid maltase 342

Acidosis 34, 159

Acquired monosaccharide intolerance 1343

Acyl coenzyme A 1339

Acrodermatitis enteropathica 876

ACTH 1035

Acute idiopathic thrombocytopenic purpura 133

Acute salt loading 869

Adams, F. H. 1082

Adenine phosphoribosyl transferase 825

Adenosine deaminase deficiency 885

Adenylate cyclase 1383

Adenylyl cyclase 291

Adolescent 709, 943, 1379

Adrenal cortex 99

Adrenal gland 1367

Adrenergic agonists 203

Adrenergic blocking agents 203

Adrenoleukodystrophy 21

Agarose technique 242

Age-race distribution 194

Aggregation 142

Ahmad, F. 4

Air temperature 1186

Air water content 1186

Akaboshi, I. 1367

Ákerblom, H. K. 339

Alam, S. 1142

Albumin 64, 785, 1360

Aldini, R. 1222

Aldosterone 39, 304, 765, 894, 1035

Aldosterone, plasma 99

Aldrin epoxidase 282

Allan, L. 896

Allen, P. L. 1173

Alloisoleucine 854

Alpha hemolytic streptococci 308

Alpha-thalassemia-2 266

Alveolar macrophage 715

Alveolar permeability 314

Amiloride 1245

Amino acid 1272

Amino acids 1142

Aminoaciduria 218

Amir, T. 1238

Amniotic fluid 353, 891

Amniotic fluid cells 224, 825, 1199

Amylase 18

$\alpha$-Amylase isoenzymes 1168

Anand, R. 203

Anand, S. K. 1118

Andria, G. 812

Androgens 67

Andujo, 0.891

Anemia 133

Angelini, C. 1379
Angiotensin 99, 183, 304, 765

Angiotensin II 1353

Annat, G. 894

Anserine 269

Antibodies 1086

Anticoagulent therapy 128

Antidiuretic hormone 1234

Anti-DNA antibodies 858

Antiplatelet therapy 128

Apnea 354, 1230

Apoproteins 757

Arachidonic acid 1073

Arakawa, K. 1230

Arant, B. S., Jr. 1204

Arcinue, E. L. 1216

Arterial oxygen content 1047

Arylsulfatases 1347

Ashkenazi, A. 776

Ashwal, S. 1104

Asphyxia 286, 357, 905, 935

Athanasiou, K. 336

Atkatsuki, J. 133

ATPase 769

Auditory brainstem response 154, 159

Aula, P. 1199

Auricchio, S. 812

Autoimmune diseases 858

Autonomic nervous system 232, 809

Avery, M. E. 1122

Aznar, J. 1180

$B_{1}$ and $B_{2}$ receptor mimetics 1067

Bacterial colonization 308

Baden, H. P. 1347

Baldassare, A. R. 1135

Balistreri, W. F. 943

Ballard, P. L. 122

Baltakse, V. 300

Barak, Y. 1026

Barbara, L. 1222

Barbero, G. T. 872

Barkowsky, W. 885

Barnett, E. V. 830

Barnhart, B. T. 1367

Bartsocas, C. 336

Bartter's syndrome 1395

Barzen, K. A. 1398

Batshaw, M. L. 1311

Battaglia, F. C. 147, 1047

Battistelli, P. A. 1379

Baylen, B. G. 1332

Baylin, S. B. 921

Bazzoli, F. 1222

Beale, E. F. 314

Beat-to-beat blood flow 1053

Beaudet, A. L. 21

Becker-McKenna, B. 183

Behavioral states 911

Bellanti, J. A. 82, 1381, 1382

Benderli, A. 1395

Ben-Galim, E. 854

Berdischewsky, M. 830

Berg, D. 1324
Berger, M. 128

Bergman, B. 1067

Bergstrom, W. H. 216

Berne, C. 1192

Berninger 1263

Bertrand, J. 819

Beta-adrenergic drugs 278

Beta-adrenergic stimulation 261

Betamethasone 326

Betamethasone therapy 122

Betke, K. 1324

Better, O. S. 1395

Bicarbonate, 18, 839

Bidani, A. 183

Bidlingmaier, F. 39

Bienvenu, F. 1040

Bier, D. M. 854, 1320

Bile acids 1222

Bile duct 740

Bile salt 943,1328

Biliary atresia 740

Bilirubin 1363

Binding, ${ }^{125}$ I-insulin 4

Bioassay 1263

Blachar, Y. 1026

Bläckberg, L. 1387

Blaufox, M. D. 304

Blood 1

Blood flow 1356, 1370

Blood volume 1324

Body posture 1403

Body water 1122

Bonderman, P. W. 103

Bone marrow megacaryocyte 1071

Bony orbital structures 703

Borer, R. C., Jr. 899

Borut, T. C. 242

Boughten, J. M. 4

Bourgeois, J. 1040

Boxerbaum, B. 715

Brain 844

Brain cell culture 1226

Branched-chain amino acids 1029

Brandt, N. J. 1029

Branski, D. 8, 803

Brasel, J. A. 1299

Breast milk jaundice syndrome 1328

Breathing frequency 1403

Brehier, A. 834

Breslow, J. L. 964

Broulard, B. H. 128

Brun, D. 113

Brush border enzymes 803

Brush border membrane 812

Brusilow, S. W. 1316

Bryant, R. C. 352

Burgio, G. R. 1111

Burkart, T. 1226

Bush, P. J. 1115

Caerulein 18

Calcagno, P. 799

Calcitonin 218 
Calcium 769, 1118

Calvin, R. T. 1343

cAmp 863, 1234

$\mathrm{CaNa}_{2}$ EDTA 330

Carbamylcholine 1398

Carbohydrate 872

Carbohydrate metabolism 209, 849

Carcinoembryonic antigen 187

Cardiac output 232

Carlisle, S. K. 4

Carlson, C. V. 1367

Carnitine 1127, 1379

Carnosinase deficiency 269

Carnosinuria 269

Carr, L. 291

Carson, B. S. 147

Cash, R. 1186

Cassady, G. 1186

Cassino, R. J. J. 1212

Cavallo, T. 128

Celiac disease 812

Cell electrophoresis 138

Cell membrane 1269

Cell metabolism 339

Central nervous system 1276

Central nervous system defects 703

Centronuclear myopathy 1196

Cerebral blood flow, total and regional 1104

Cerebral spinal fluid 1276

Cerebroside sulfate loading test 224

Cerebroside-sulfotransferase 1226

Cerebrospinal fluid 286

Chaimovitz, C. 1395

Chalmer, B. 1139

Chalmers, R. A. 1097

Chan, G. M. 1320

Chang, C. H. 1216

Chartrand, L. 1360

Chase, G. 194

Chediak-Higashi syndrome 901

Chemotaxis 142, 1147

Chenodeoxycholic acid 1222

Chernick, V. 357

Chesney, R. W. 209

Chia, D. 830

Cholesterol 24, 64, 113, 194, 272

$\left[{ }^{14} \mathrm{C}\right]$ Cholesteryl lignocerate 21

Cholic acid 943, 1222

Choline 47, 899

Choroidal blood flow 1047

Chow, D. 1035

Choy, F. Y. M. 54

Christensen, B. 194

Christensen, E. 1029, 1412

Christensen, R. A. 203

Christensen, R. D. 806, 1147

Chrzanowski, B. L. 1356

Churchill, P. C. 183

Ciani, A. 782

$\mathrm{Clq}$ binding activity 858

Cier, J. F. 894

Ciliary resorption 1173

Circadian patterns 345

Circulating immune complexes 830

Citric acid cycle 1127

Clark, C. M., Jr. 291

Clark, J. B. 291

Cochlea 159

Cocks, T. 28

Coeliac disease 776

Cohen, T. 963
Cohens, S. 1276

Coicadan, L. 109

Colle, E. 209

Coltina, A. 1222

Columnar cell 1173

Computer-identified breathing pauses 1230

Concentrating capacity 1234

Conditioned medium 261

Continuous positive airway pressure 1132

Contreras, G. 272

Copper 1040

Cord blood 4

Cord serum 757

Cortical nephrons 799

Corticosterone 39

Cortisol 39, 122, 1367

Cortisone 39

Costeloe, K. 1053

Cottreau, D. 1162

Coulombe, P. 228

Coulter, D. M. 1122

Cowan, R. H. 1204

Cowett, R. M. 1391

Creatine kinase 935

Crescent formation 128

Crokaert, R. 1088

Cros, J-C. 793

Cryoglubulins 858

Csaba, I. F. 765

Cucchiara, S. 812

Cuestas, R. A., Jr. 935

Cultured amniotic cells 749

Cummiskey, J. 762

Cunningham, R. J., III 128

Cyclic adenosine 3':5'-monophosphate 261

Cyclic nucleotides 218

Cystic fibrosis 118, 187, 261, 353, 715, 830, $863,921,1086,1088,1168,1173,1212$, $1245,1263,1269,1360,1387,1398$

Cystine 785

Cystinosis 785

Cystinuria 109

Cytosol membrane 812

Dab, I. 1088

Dagoglu, T. 216

Dakshinamurti, K. 896

David, M. 819

Davidson, R. G. 54

Davis, P. B. 83, 863

Dearborn, D. G. 715, 1245

de Groot, G. W. 896

de Groot, I. 194

Dehydroepiandrosterone sulfate (DHAS) 1367

DeLennoy C. W. 926

Delivoria-Papadopoulos, M. 1349

Demers, L. M. 190

Demko, C. A. 715

Demattsy, V. 47

Denaro, M. 1086

11-Deoxycorticosterone 39

deRitis, G. 812

Deschamps, I. 300

Desjeux, J. F. 109, 300

Despland, P. A. 154, 159

Dessart, Y. 894

Development 703, 1111, 1192, 1226, 1272 , 1304, 1349, 1356, 1374, 1379

DeVizio, B. 812

Dexamethasone 899
Diabetes 47, 1192

Diagnostic technique 1115

Diamine oxidase 118,921

Diarrhea 839, 1382

Dicarboxylic aciduria 1097

Dicetylphosphate 24

Dickerman, J. D. 1139

Diet 272, 1040

Dietary cholesterol 1061

Dietary fat 1061

Dietary hypocalcemia 218

Digestive peptidases 812

1, 25-Dihydroxyvitamin $D_{3} 330$

Dillon, M. H. 1212

Dipalmitoyl-PC 24

Dipeptides 1272

di Sant'Agnese, P. A. 83

Distended pulmonary artery 1332

DNA 88, 336

DNA polymerase 881

Dodge, M. E. 1320

Donzelli, F. 1379

Dopamine 18

Dopamine $\beta$-hydroxylase activity 894

Dorfman, A. 1023

Dorner, R. W. 1135

Dörr, H. G. 39

Doughty, R. A. 858

Douglas, W. H. J. 899

Down's Syndrome 88, 336

Doxiadis, S. A. 947

Dreyfus, J. C. 342,1162

Drukker, A. 304

Drummond, K. N. 209

Drummond, W. H. 1311

Dubois, J. D. 247

Duchenne muscular dystrophy 1196

Ductus arteriosus 1073

Ductus venosus 1

Dupont, C. H. 209

Dussault, J. H. 228, 247

Edelmann, C. M. 304

Edwards, W. H. 286

Egan, E. A. 314

Eguren, L. 1127

Eisner, G. 799

Ekblom, P. 1199

Electrical impedance plethysmography 1053

Electrolytes 765, 1035, 1260, 1343

Eliam, E. 963

Elrad, H. 216

Emmanouilides, G. C. 1332

Encounter rates 1115

Endotoxin 939

Entero-amino acid transport system 109

Enterocyte membrane 109

Enterohepatic circulation 1222

Enzyme 1356

Enzyme deficiencies 956

Enzyme kinetics 729

Enzymes 939

Enzyme, structurally altered 54

Epinephrine 291, 916

Epithelial lining 740

Epstein, J. 964

Epstein, M. A. F. 232, 809

Epstein, M. F. 47, 278

Epstein R. A. 232, 809

Eriksson, U. 1192

Ermocilla, R. 1186 
Ertl, T. 765

Erythrocyte 4, 1269, 1349

Erythrocytes 729, 825, 1196

Escobedo, M. B. 103

Estelles, A. 1180

Estrada, P. 18

Etzioni, A. 1395

Evans, G. W. 876

Exocrine cells 1398

Experimental infection 1408

Extracorporeal therapy 113

Faden, H. 803

Faden, H.S. 8

Fat digestion 1360

Fatty acids, 296, 1088, 1328

Faulkner, G. T. 722

Feather, M. S. 872

Feig, S. A. 96, 1168

Feldman, G. L. 153, 1413

Felice, A. E. 266

Fennessey, P. V. 12

Fernandez, M. 272

Festi, D. 1222

Fetal 203

Fetal erythrocytes 138

Fetal glucose $/ \mathrm{O}_{2}$ quotient 147

Fetal hypoxia 1071

Fetal metabolism 103, 147

Fetal pulmonary maturity 722

Fetus, 47, 67, 93, 236, 360, 749, 782, 793, 825, $891,905,939,947,1047,1067,1104$. 1162,1379

Fetus/neonate 1250

Fewell, J. E. 1132

Fibrinolysis 1180

Fibroblast 896

Fibroblast culture 956

Fibroblasts 21, 67, 88, 261, 785, 863, 899, 932 . 1199,1339

Fibroblasts, human 118

Fischer, G. W. 142

Fischer, T. J. 242

Fiser, R. H. 1320

Fisher, D. A. 99,869

Fisher, D. E. 926

Flamenbaum, W. 799

Flavoprotein 1339

Fleischman, A. 1276

Fleischmann, L. 183

Fleisher, L. D. 269

Fluharty, A. L. 224, 890

Flux, M. 1398

Forest, M. G. 819

Forster, E. 1035

François, R. 894

Frank, S. 1260

Frantz, I. D., III 47

Fredrikzon, B. 1387

Free fatty acids 1088

French, J. 1276

Friedman, Z. 190

Fructose 103

Full-term newborn 1180

Furosemide 765, 869, 1118, 1353

Gagne, C. 113

Gahl, W. A. 118

Galactos 1-phosphate uridylyl transferase 729

Galactosemia 729

Galambos, R. 154, 159
Galey, W. R. 1269

Gall, D. G. 250

Galy, G. 1040

Gamma globulin 788

Gamma radiation 336

Ganguli, S. 203

Gard, S. E. 242

Garg, G. 1328

Garson, A., Jr. 881

Gartside, P. S. 272

Gastric inhibitory polypeptide 300

Gaucher's disease 54

Gaull, G. E. 735

Genetics 1347

Genetic variant 269

Gentamicin 1204

Gershon, A. A. 885

Gestation 47

Giacomo, M. 147

Gilabert, J. 1180

Gilfoil, M. 128

Gilland, B. K. 1168

Gillespie, C. T. 187

Gillette, P. C. 881

Gillon, M. J. 1269

Ginns, E. 1276

Glover, T. W. 88

Glucagon 103, 216, 1320

Gluck, L. 1250

Gluckman, P. D. 122

Glucocorticoids 39

Gluconeogenesis 793, 926

Glucose 28, 103, 296, 782, 926, 1142, 1343, 1391

Glucose homeostasis 1320

Glucose intolerance 50

Glucose metabolism 96

$\beta$-Glucosidase 54

Glueck, C. 194

Glueck, C. J. 272

Glutaric aciduria type II 12, 1339

Gluten sensitivity 776

D-Glyceric acidemia 1029

Glycine 932

Glycine pool size 1238

Glycine turnover rate 1238

Glycolytic enzymes 1349

Glycoprotein 187

Gml ganglioside 1276

Goblet cell 1173

Godoy, R. R. 1374

Golden, J. 1186

Goldman, S. L. 50

Goldsmith, D. I. 304

Goldstein, M. A. 881

Goodman, S. I. 12

Gordon, B. A. 1411

Gore, I. 1304

Gotoff, S. P. 788

Gottesdeiner, K. 1035

Graebe, H. 729

Graham, S. P. 881

Granulocytes 901

Granulopoietic factor(s) 1026

Grasset, E. 109

Grasso, S. 782

Gravely, M. E. 266

Gresham, E. L. 103

Gross, I. 834

Group B streptococcal immune globulin 788

Group B streptococci 788, 1408
Growth disorder 339

Growth hormone 122, 339

Growth retardation 1071

Grumbach, M. M. 122

Guanyl nucleotides 291

Guerra, G. 1374

Guillemonault, C. 762

Gjory, E. 765

Haas, B. 825

Häberle, D. A. 1234

Haddad, G. G. 232, 809

Hadorn, B. 261

Hallman, M. 326, 1250

Halton, D. M. 932

Hamilton, J. R. 250

Hamosh, M. 93

Hamosh, P. 93

Handzel, Z. T. 776

Hansen, T. L. 1412

Hanson, N. 911

Harper, R. 1230

Harper, R. M. 345

Harries, J. T. 1382

Hartig-Beecken, I. 278

Hartlage, P. L. 224

Hadorn, B. 261

Hatch, T. F. 8, 803

Haust, M. D. 1411

Haworth, J. C. 896

Haymond, M. W. 854

HbG-Philadelphia 266

HbS-Philadelphia 266

Heald, F. P. 1061

Hearing loss 154

Heart 291

Heart rate variability 232, 809

Heath, M. F. 254, 846

Hedner, T. 1067

Heiner, D. C. 60

Heiss, G. 194

Hellkuhl, B. 729

Hematocrit 1324

Hematuria 128

Hemli, S. 849

Hemolytic assay 1135

Henderson, T. 96

Heparin-agarose 113

Hepatic encephalopathy 844

Heptner, W. 300

Herschkowitz, N. 749, 1226

Herzberg, V. L. 4

Herzfeld, A. 1304

Heterozygote 921

Heubi, J. F. 943

Heyman, M. 109

Heymann, M. A. 1311

Hidden 19S IgM rheumatoid factor 1135

Higginbottom, M. C. 703

Hill, D. E. 4

Hill, H. R. 806, 1408

Hill, S. C. 863

Himulkai, M. 1272

Hirata, T. 50

Hirschhorn, R. 885

HMG-CoA reductase 1061

Ho, C-K. 224

Hodgman, E. 1230

Hodgman, J. E. 345

Hoffmann, K. 1226

Hollingsworth, M. 916 
Homozygote 921

Homozygous familial hypercholesterolemia 113

Hooker, P. A. 1347

Hopfer, U. 1245

Hoppenbrouwers, T. 345, 1230

Horner, S. R. 1139

Horton, W. A. 966

Hoshi, T. 1272

Hubbard, V. S. 968

Huisman, H. J. 266

Human amniotic fluid 722

Human chorionic gonadotropin 819

Human milk 876

Human placenta 769

Hunter, K. W. 142

Hydrogen ion 709

$7 \alpha$-Hydroxylase 1061

17-Hydroxyprogesterone 39

25-Hydroxyvitamin D 360

Hyperammonemia 1316, 1370

Hyperbilirubinemia 947

Hypercapnea 34

Hypercapnic ventilatory response 762

Hypergammaglobulinemia 128

Hyperglycinemia 1029

Hyperinsulinemia 147

Hypersarcosinemia 12

Hypertension 1035

Hyperviscosity 1320

Hypocalcemia 216

Hypocomplementemia 858

Hypoglycemia 1097

Hyperglycinemia 1029

Hypoglycine metabolite analogue 1097

Hypothalamus-pituitary-thyroid axis 228

Hypoxanthine 905

Hypoxanthine-guanine phosphoribosyl transferase 825

Hypoxemia 34

Hypoxia 1104

Hypoxic ventilatory response 762

Ichthyosis 1347

Idar, D. 776

Idiopathic Fanconi Syndrome 209

IgD 60

$\operatorname{lgE} 60$

Ikegami, M. 1082

lleum 839

Immune complexes 858

Immunodeficiency 242

Immunodeficiency diseases 780

Immunoglobulin-containing cells 953

Immunologic characterization 1162

Implantation 308

Indomethacin 1395

Ineichen, E. 272

Infant 236, 885, 943

Infants 809

Infection 308

Ingleson, L. D. 834

Insulin 4, 28, 50, 103, 147, 216, 300, 782, 834, 1320, 1391

Insulinopenia 209

Intermittent positive pressure ventilation 1186

Intestinal absorption 1272

Intestinal mucosa 953

Intestinal $\mathrm{Na}$ transport 250

Intestinal permeability 250

Intestinal solute load 1343
Intestinal transport 1142

Intracellular water 1122

Intragastric lipolysis 1360

Intrauterine growth retardation 1356

Isoenzyme 935

Isoleucine 854

Isoproterenol 261, 863, 872

Isotopic dilution technique 943

Jacobson, M. S. 1061

Jacobson, W. 254, 846

Jamaican vomiting sickness 1097

Jayachar, S. 1111

Jejunum 839, 1382

Jengo, J. A. 1332

Jensen, D. K. 345

Jeune, M. 819

Jiminez, J. M. 891

Jobe, A. 319

Jobst, K. 138

John, E. 1186

Johnson, J. J. 891

Johnson, J. P. 722

Johnson, P. E. 876

Jones, J. G. 780

Jones, K. L. 703

Jones, M. D., Jr. 1047

Joppich, R. 1234

Jori, G. 1363

Jose, P. 799

Jukkola, A. F. 1204

Juratsch, C. E. 1332

Juvenile rheumatoid arthritis 1135

Juxtamedullary nephrons 799

$\mathrm{K}^{+}$efflux 1398

Kahn, A. 1162

Kanjanapone, V. 278

Kaplan, B. S. 209

Kaplan, S. L. 122

Katsuki, T. 1370

Kelly, P. D. 788

Kempe, C. H. 1155

Kennedy, J. R. 1173

Kerian, A. 1118

Kerr, D. 28

Keto-enol tautomerization 854

$\alpha$-Ketoglutarate 1316

Ketonuria 209

Khan,, M. A. 1061

Kidney 190, 926, 1118, 1132, 1234, 1260, 1395

Kidney failure 183

Kiefer, C. A. 780

Kihara, H. 224, 890

Kilbourn, J. P. 259

Kipouros, K. 947

Kjellmer, I. 905

Klein, R. B. 242

Klein, W. 96

Klish, W. J. 1343

Knorr, D. 39

Köferl, F. 1324

Kohan, M. 1212

Kokuburn, Y. 133

Kolodny, E. H. 849

Kølvraa, S. 1029

Konno, T. 1272

Kooh, S. W. 360

Kosztolanyi, Gy. 138

Krantz, B. 1356

Krasner, J. 8, 803
Kretchmer, N. 1093

Kretchmer, R. R. 788

Krieger, H. 1324

Krieger, I. 932

Kuckenbrod, P. J. 715

Kugler, J. D. 881

Kukilus, J. 1347

Kumar, S. P. 1349

Kurlandsky, L. E. 1263

Kwashiorkor 1260

Kwiterovich, P. 194

Lactic acidosis 956

Lafferty, J. L. 1403

Laks, M. M. 1332

Lamellar body 846

Lamellar body fraction 722

Lane, D. M. 757

Lang, A. 729

Lanzavecchia, A. 1111

Lapidot, A. 1238

Lasalle, R. 1360

Laurila, P. 1199

Law, H. S. 232

Lawson, A. M. 1097

Lawson, E. E. 357

Lazzari, R. 1222

Lead 330

Leake, R. D. 869, 1320

Lebenthal, E. 8, 803, 1356

Lebowitz, J. L. 1245

Lecithin 899

Lecoq, A. 819

Lectins 1199

Lee, S. K. 60

Leidy, G. 308

Leisti, S. 339

Leistner, H. L. 809

Lemons, J. A. 103

Lemons, R. 785

Le Provost, E. 793

Lesch-Nyhan Syndrome 825

Lestradet, H. 300

Leucine 782

Leukemia 133

Leukocyte 1370, 1408

Leukocytes 342

Levan, N. E. 1374

Levin, S. 1026

Levi, Y. 1395

Levine, L. S. 1035

Levitsky, L. L. 926

Leydig cells 819

Lieberman, E. 1118

Liebers, I. 749

Lifshitz, F. 1142

Liggins, G. C. 122

Linderkamp, O. 1324

Lingual lipase 1360

Lipase 1328, 1387

Lipid 872

Lipid biosynthesis 1226

Lipids 296, 757, 1387

Lipoprotein 64, 272

Lipoproteins 113, 757

Liposome 254

Liposomal phosphatidylcholine 24

Lithocholic acid 1222

Liver 1, 24, 236, 735, 939, 1238, 1304

Liver enzymes 1216

Li Volti, S. 1196 
Lockwood, D. H. 921

Longo, G. 1196

Longo, L. D. 1104

Lou, M. 113

Lowe, C. 34

L-Thyroxine $\left(T_{4}\right) 228$

Luengas, J. 1260

Lundborg, P. 1067

Lung, 24, 34, 254, 282, 296, 314, 319, 830, 834, $846,872,916,1082,1192,1212,1304$

Lung epithelial cells 899

Lung maturation, 93, 1067

Lung surfactant 1250

Lupien, P-J. 113

Luteinizing hormone 819

Lütschg, J. 64

Lymphocytes 336, 342, 901

Lymphoid cell 1370

Lysine 109

Lysosomal enzyme activities 901

Lysosomal storage disease 1199

Mace, J. W. 12

Machinot, S. 300

Macri, C. N. 1212

Macrophages 1212

MacSween, J. M. 187

Madansky, D. L. 357

Maes, M. 67

Magnesium 769

Majcher, J. S. 1104

Malnutrition 28

Maniscalco, W. M. 834

Mantagos, S. 1339

Maple Syrup Urine Disease 854

Marasmus 1260

Markowitz, M. E. 330

Maroteaux, P. 965

Martin, T. R. 722

Martinez, J. R. 18, 872

Marton, L. J. 921

Matsuda, I. 779, 1370

Matthews, D. E. 854

Maturation 780, 1040

Mawhinney, T. P. 872

Mayson, S. M. 266

Mazza, N. M. 232

Mazzacca, G. 812

Mazzella, G. 1222

McAfee, J. 1127

McCabe, E. R. B. 12

McConathy, W. J. 757

McCullough, R. 762

McDevitt, M. 1186

McGeeney, K. F. 969

McInnes, R. R. 209, 218

McVicar, M. 1142

Mease, A. D. 142

Meberg, A. 1071

Meconium aspiration syndrome 34

Mehendale, H. M. 282

Meier, C. 749

Mellins, R. B. 232, 809

Melnick, V. L. 1295

Membrane maturation 138

Mendoza, S. 272

Mercuric chloride 183

Meschia, G. 147

Metabolic acidosis 286, 839
Metabolic rate 28

Metabolism 50, 67, 269, 282, 296, 319, 360, $749,785,885,896,926,943,956,1035$, $1061,1127,1238,1349,1387,1391$

Metabolism, amino acids 932

Metabolism, drug 939

Metabolism, glucose 793

Metabolism, glycine 1029

Metachromatic leukodystrophy (MLD) 224

4-Methylumbelliferylguanidinobenzoate 353

Metrakas, J. D. 60

Meyshens, F. L. 780

Michels, V. V. 21

Migeon, B. R. 67

Migeon, C. J. 67

Migration inhibition factor 776

Miller, A. 266

Miller, A. L. 849

Minami, R. 236

Mineralocorticoids 39

Miranda, R. 1374

Mitchell, R. A. 1216

Mitochondria 735, 1216

Mitochondrial inhibitors 769

Mollica, F. 1196

Momma, K. 1073

Monocyte chemotaxis 242

Mononuclear cells 96

Moore, T. L. 1135

Moorehead, H. C. 103

Moorjani, S. 113

Mordasini, R. 64

Morin, C. L. 1360

Morrison, J. A. 272

Morselli, A. M. 1222

Mortality 1139

Mortola, J. P. 1403

Morton, D. 18

Mowery, R. 194

Mrozinska, K. 1142

Mucopolysaccharides 749

Mucopolysaccharidosis II (Hunter) 749

Mucus-stimulating substance 1263

Muller, D. P. R. 1382

Mullon, D. K. 93

Muñoz, R. 1260

Murphy, S. 1269

Muscle 1374

Mutagenesis 88

Myasthenia gravis 1196

Myocardial chemical sympathectomy 881

$\mathrm{Na}^{+}$balance 304

$\mathrm{Na}^{+}$transport system 932

Nadler, H. L. 353

Nagata, N. 779, 1370

Nair, P. P. 1061

Nakamura, K. 236

Nakao, T. 236

$\left(\mathrm{Na}^{+}-\mathrm{K}^{+}\right)$-ATPase 1382

$\mathrm{Na}^{+} \mathrm{K}^{+}$ATPase activity 844

Naloxone 357

Nattie, E. E. 286

Naseem, S. M. 1061

Natural antibodies 1111

Nelson, D. A. 1078

Nelson, R. M. 314

Németh, M. 765

Neonatal adrenal function 326
Neonate 232, 308, 357, 793, 894, $911,926,935$, $939,947,953,1026,1040,1047,1053$, $1071,1122,1132,1147,1204,1230$, $1276,1320,1332,1349,1374,1391$

Neonates 765

Nephrotic syndrome 1078

Nephrotoxicity 1204

Neurological damage 935

Neutrophil 142, 1147

Neutrophilia 1026

Neutrophils 806, 1212

Neutrophil storage pool 806, 1147

Neutropenia 806

New, M. I. 1035

Newborn 1, 12, 50, 93, 142, 154, 159, 190, 360 , 880

Nichols, B. L. 1343

Nishihara, S. 1073

Nitzan, M. 1356

Nolen, P. A. 103

Nonestified fatty acids 1088

Nooman, M. E. 858

Nonketonic hyperglycinemia 932

Nordling, S. 1199

Norepinephrine 881

Norton, J. B., Jr. 1132

Nucete, H. 272

Obesity $\mathbf{3 0 0}$

Oetliker, O. H. 64

Oh, W. 1320, 1391

Ohsuka, H. 1370

Okken, A. 911

$\left[{ }^{14} \mathrm{C}\right] \mathrm{Oleate}$ glycerol 21

Optic vesicle 703

Or, A. 776

Organic aciduria 12

Organ culture 834

Organs 1324

Ornithine transcarbamylase 1370

Ornithine transcarbamylase deficiency 735

Osang, M. 825

Osher, A. B. 830, 1082

Osmolality 1343

Osmotic water permeability 1269

Otitis media 1115

Oto, Y. 1073

Oulton, M. 722

Oxotremorine 916

Oyanagi, K. 236

Padnos, D. M. 788

Paez, P. C. 1349

Pallotta, J. A. 849

Palumbo, G. 782

Pancreas 8, 18, 1168, 1356, 1391

Pancreatic hormone secretion 203

Paniagua, R. 1260

Paradis, Y. 1360

Parathyroid hormone 218, 330

Parker, A. 18

Parkhill, T. 1353

Parks, J. K. 1127

Parrilla, J. J. 1180

Passive immunization 788

Paton, J. B. 926

Pavone, L. 1196

Peeters, L. L. H. 1047

D-Penicillamine 330 
Pepsin 709

Parenteral nutrition 50

Perheentupa, J. 339

Periocular structures 703

Peripheral hearing disorder 159

Perkkiö, M. 953

Petmezaki, S. 947

Petrusick, T. 128

Phagocytosis 715

Phenobarbital 947

Phenylketonuria 849

Philipps, A. F. 147

Phosphatidic acid phosphohydrolase 891

Phosphatidylcholine 254, 916

Phosphaturia 218

Phosphofructokinase 1162

Phospholipase A 254, 846

Phospholipid 722

Phospholipid biosynthesis 1250

Phospholipids 47, 296, 319, 326, 334, 891, 1192

Phototherapy 1363

Phytohemagglutinin 142

Picolinic acid 876

Picon, L. 793

Picou, D. 28

Pilocarpine 18, 872

Pitot, H. C. 118

Pituitary-thyroid axis 247

Pivetta, O. H. 1212

Placenta 228

Plasma renin concentration (PRC) 304

Plasma volume 1324

Plasminogen 1180

Plasminogen amino acids 1180

Platelets 1078

Platelet volume 133

Plebani, A. 1111

Poenaru, L. 342

Poland, R. L. 1328

Pollack, M. 830

Polyamines 339, 921, 1196

Polycythemia 1071

Pompe's disease 342

Portal-systemic encephalopathy 1316

Portha, B. 793

Postenteritis syndrome 1382

Postnatal development 250

Preferential inhibition 54

Premature 50, 1082

Prenatal diagnosis 224, 353

Pressure-volume relationship 1067

Preston, B. 1374

Procaine penicillin 1139

Progesterone 39

Progestins 39

Prolactin 93, 122

Propionyl CoA 1127

Prorenin 1353

Prostaglandin 1073, 1395

Prostaglandin $E_{1} 863,1311$

Prostaglandin indoperoxide formation 1078

Prostaglandins 765

Prostaglandin synthetase 190

Proteases 353

Protein 872

Protein energy 28

Proteinuria 128

Proteinuric Nephrotic Syndrome 64

Proteolysis 785
Pseudo arylsulfatase A deficiency 224

Pseudomonas 715

Pseudomonas aeruginosa 830

Pulmonary morphology 1186

Pulmonary phospholipids 278

Pulmonary vasoconstriction 1311

Purkiss, P. 1097

Quissell, D. O. 1398

Rabin, D. L. 1115

Rachelefsky, G. S. 242

Radioimmunoassay 60, 1222

Rahm, P. 825

Raivio, K. O. 326, 1199

Ram, M. L. 1216

Ranis, M. B. 296

Rao, G. J. S. 353

Raper, S. M. 1304

Rapidly progressive glomerulonephritis 128

Rapisoda, A. 1196

Rassin, D. K. 269

Rate of weight gain 28

Rauh, W. 1035

Rebenthal, E. 8

Receptors, insulin 4

Red cell mass 1324

Redman, W. 308

Reisner, S. H. 1238

Reitano, G. 782

Renal failure 128

Renal function 1204

Renin 183, 765, 869, 894, 1035, 1353

Renin, plasma 99

Rennert, O. M. 1398

Reovirus type 38,803

Reserpine 18, 872

Respiration 1403

Respiratory distress syndrome 93, 122, 254, $278,326,722,916,1082,1192$

Respiratory rates 345

Respiratory variables 1230

Retinal blood flow 1047

Reye's like syndrome 1097

Reye's syndrome 735, 1216

Reynolds, J. W. 1367

Rhead, W. 1339

Riegel, K. P. 1324

Riesen, W. 64

Rifkind, B. 194

Rigal, D. 1040

Rimoin, D. L. 966

Robillard, J. E. 839

Robinson, B. H. 956

Robinson, H. M. P. 28

Roda, A. 1222

Roda, E. 1222

Rogiers, V. 1088

Rolfe, P. 1053

Romeo, G. 1086

Rooney, S. A. 834

Rosenfeld, C. R. 891

Rosenstein, B. J. 921

Roscher, A. A. 261

Rosen, J. F. 330

Rosenberg, I. 776

Rossi, E. 1363

Rothstein, G. 806, 1147

Rothwell, S. W. 67
Roulet, M. 1360

Roy, C. C. 1360

Rubaltelli, F. F. 1379

Rudolph, A. M. 1311

Rugolo, S. 782

Ruymann, F. B. 142

Saenger, P. 1035

Saline loading 304

Saliva 1245

Salivary secretion 1168

Salmon, M. 947

Salwen, H. R. 269

Sama, C. 1222

Sana, L. 1040

Santos, D. 1260

Santos, J. I. 1408

Saralasin 1353

Sarcosinuria 12

Sassard, J. 894

Saugstad, Ö. D. 905

Savilahti, E. 953

Scanlon, J. W. 93

Schaffner, F. 735

Schaub, J. 825

Schmid, A. J. 261

Schoentgen, S. 1374

Schrader, J. 1234

Schreiner, R. L. 103

Schultz, G. E. 1328

Schwartz, R. 1391

Scriver, C. R. 209, 218

Seale, T. W. 1398

Secretory IgA 1111

Segal, D. J. 88

Sepsis 806

Sera 60

Serine 932

Severe combined immunodeficiency 885

Sex-specific distribution 194

Shaffer, T. H. 34

Shapiro, J. M. 735

Sharp, M. J. 899

Sheldon, R. E. 1047

Shenkman, L. 885

Sheperd, R. W. 250

Sherwood, W. G. 956

Shigeoka, A. O. 806, 1408

Shin-Buehring, Y. S. 825

Short-chain fatty acid 844

Sideris, E. G. 336

Siegel, S. 1353

Siegel, S. R. 99, 869

Siegrist, H. P. 1226

Siimes, M. A. 339

Sills, R. H. 1078

Silver, H. K. 1151

Simbala, J. A. 1168

Simmons, M. A. 147

Single nephron filtration 799

Sippell, W. G. 39

Sivieri, E. M. 34

Skin 1374

Sleep apnea 762

Sleep state 232, 345, 809, 911

Small intestine 812, 1304

Smith, D. W. 703

Smith, Y. F. 93

Sodium balance 1395 
Sodium taurochalate 54 Sodium transport 1245 Sogawa, H. 236

Sonawane, B. R. 939

Sordelli, D. O. 1212

Sperling, M. A. 203

Spermine 1196

Spitz, L. 740

Spitzer, A. 304

Spitzer, R. E. 1078

Sprunt, K. 308

Spycher, M. A. 749

Stanescu, D. V. 965

Stanescu, R. 965

Starvation, maternal 103

Steatorrhea 943

Sterman, M. B. 345,1230

Steroid sulfatase 1347

Stewart, R. M. 849

Stiehm, E. R. 242

Stinson, D. 722

Stomach 709

Stonestreet, B. 1391

Storch, S. 96

Streptococci 806

Streptococcus pneumoniae 1139

Stuart, M. J. 1078

Stumpf, D. A. 1127

Submandibular gland 1398

Succinate: CoA ligase 1127

Sudden Infant Death Syndrome 345, 762

Sulfolithocholylglycine 1222

Sultan, C. 67

Sulyok, E. 765

Surfactant $254,278,314,319,326,722,834$, $846,916,1082$

Susa, J. B. 1391

Sympathetic nervous system 894

Systemic arterial pressure 799

Systemic lupus erythematosus 858

Talamo, R. C. 1263

Tallan, H. H. 735

Tamir, I. 194

Tanaka, K. 1339

Tanaka, K. R. 60

Tanaka, T. 901

Tandler, B. 715

Tarascio, A. 1347

Tavani, N., Jr. 799

Taylor, A. 1245

Taylor, J. 956

Taylor, W. H. 709

Tényi, I. 765

Teramoto, K. 326

Terashima, T. 799

Terminal deoxynucleotidyl transferase $\mathbf{7 8 0}$

Termino, G. 782

Terry, M. L. 296

Testosterone 819
Theophylline 1226

Thibeault, D. W. 1332

Thiringer, K. 905

Thoene, J. G. 785

Thomassen, M. J. 715

Thompson, H. K., Jr. 881

Thrombocytopenia 1071

Thrombocytopenias 133

Thrombopoiesis 133

Thymidine kinase activity 1304

Thymus 780

Thyroid hormone 247

Thyroxine 247

Tolerance tests 849

Tomita, E. 133

Towers, B. 1082

Tracheal epithelium 1173

Tracheal fluid 891

Tran, N. 34

Transcutaneous oxygen tension 911

Trauner, D. A. 844

Travis, L. B. 128

Travis, S. F. 1349

Triglyceride 64, 194, 272

Triiodothyronine 247

Tripp, J. H. 1382

Tripp, M. E. 1311

Trosko, J. E. 88

Trypsin 138, 1086

Tsang, R. C. 769

Tsao, F. H. C. 24

Tsay, K. K. 224

Tsukazaki, H. 236

Tydén, O. 1192

Type I lipid storage myopathy 1379

Tyrosine aminotransferase 896

Tyrosinemia 896

Udall, J. N. 1343

Uehara, I. 779

Uemura, S. 1073

Ugazio, A. G. 1111

Ulane, M. M. 863

Urea cycle enzymopathies 1316

Urea-synthesizing enzymes 236

Uremia 1142

Urn cell complex 1263

Vadnay, L. 899

Vagi nerves 1403

Vain, N. 1104

Valaes, T. 947

Valine 932

Vanella, A. 1196

Van Petten, G. R. 1

Varga, F. 765

Vascular resistance 1332, 1374

Vasodilator 1311

Vasopressin 869

Vergani, L. 1379
Vieth, R. 360

Vincent, M. 894

Vinicor, F. 291

Viral enteritis 8

Virtanen, I. 1199

Vis, H. L. 1088

Vitamin D deficiency 218

VLBW infant 50

Vogel, L. C. 788

von Figura, K. 729

Walker, P. 247

Walker, V. 709

Walker Smith, G. J. 834

Walser, M. 1316

Walsh, M. M. J. 353

Wapnir, R. A. 1142

Warburton, D. 1391

Warshaw, B. L. 1118

Warshaw, J. B. 296

Water 1343

Webber, B. B. 266

Weber, A. M. 1360

Weil, T. V. 762

Weisman, U. N. 261, 749

Weiss, T. D. 1135

Weitzman, R. E. 869

Werdnig-Hoffman disease 1196

Whitelaw, A. 1097

Whitsett, J. A. 769

Wiesmann, U. 1226

Will, P. C. 1245

Wilson, C. M. 834

Wirtz, A. 825

Wisniewski, K. 269

Wolf, B. 153, 1413

Wolf, R. O. 968

Wong, W. H. 1374

Wood, R. E. 715

Wu, P. Y. K. 1374

Yaffe, S. J. 939

Yamamoto, T. 1370

Yang, L. C. 858

Yotti, L. P. 88

Young, L. S. 830

Younoszai, M. K. 839

Zachman, R. D. 24

Zakauddin, S. 1320

Zerhouni, N. 67

Zimmet, S. 799

Zinc 876, 1040

Zink, J. I

Zlotogora, J. 963

Zuckner, T. 1135

Zwillich, C. 762

Zymogen enzymes 8 